\title{
Condition assessment and service life prediction of a marine concrete structure: an interesting case study
}

\author{
Joost Gulikers* \\ *Ministry of Infrastructure and Water Management, Rijkswaterstaat GPO, P.O. Box 2232, 3500 GE Utrecht, Netherlands
}

\begin{abstract}
On the windward side of an existing box girder bridge exposed to aerosol and incidentally spray chloride profiles were determined at an age of 18 and 32 years as to obtain data on the development of chloride ingress over time and to predict the time to onset of reinforcement corrosion. According to the chloride contents determined at the level of the reinforcing steel for an age of 18 years corrosion was likely to be present, however neither visual inspections nor large scale potential mapping revealed any spots suspect of corrosion. Chloride profiling performed at an age of 32 years indicated that chloride ingress was much slower than was anticipated. A full probabilistic approach was adopted to predict the development of chloride ingress over time and onset of reinforcement corrosion using updated values for the so-called ageing factor and the critical chloride content. However, the results obtained with these predictions proved to be far more pessimistic than is shown by the actual condition of the bridge. As a consequence it was decided not to rely anymore on predictions based on numerical models using probabilistic calculations but in contrast to adopt a pragmatic engineering approach using common sense as a basis.
\end{abstract}

\section{Introduction}

For marine concrete structures reinforcement corrosion induced by penetrating chlorides is considered the major threat for durability. Condition assessment by regular visual inspections is the common procedure adopted in practice. Generally, more in-depth investigations are considered after a reinforced concrete structure shows clear visual evidence of distress caused by corrosion of the embedded reinforcement. Such in-depth investigations may comprise non-destructive techniques e.g. potential mapping and cover depth measurements. However, the more common approach is to drill a number of cores, determine the chloride penetration profile and, based on the chloride content at the level of the reinforcing steel, judge if corrosion is likely to be present or will occur in the near future. It is frequently suggested that a pro-active strategy based on in-depth investigations performed on a regular basis, e.g. every 10 years, should be preferred as this in the long term will allow for a more cost-effective approach to maintenance and repair.

As part of a national program on condition assessment of existing concrete structures exposed to a marine environment, in 2002 a large number of concrete cores were drilled from a number of concrete structures along the Dutch coast [1]. The objectives of this research program were to collect data on chloride ingress and to validate the service life model developed in the European program DuraCrete [2]. At the time of inspection and measurements, none of the structures investigated, except one, showed any signs of damage resulting from corrosion.

A box girder bridge crossing the Eastern Scheldt was one of the structures investigated. Based on the findings obtained in 2002, during the time period 2005-2016 additional large-scale investigations were performed including potential mapping. Eventually it was decided to organise a large campaign in 2016 comprising a large series of drilled concrete cores to have an updated physical evidence of chloride ingress.

This paper presents and discusses the findings and interpretation of the chloride profile measurements obtained in 2002 and 2016 on the wall of a box girder bridge facing the North Sea (windward side).

\section{Description of the box girder bridge}

The box girder bridge is built over a storm surge barrier, see Fig. 1, and comprises 68 prestressed elements with 62 elements made from gravel concrete whereas the remaining 6 bridge elements $(3 \times 2$ abutments $)$ have been constructed in lightweight concrete. All gravel concrete bridge elements have a span of $45 \mathrm{~m}$ and the concrete composition used is based on $325 \mathrm{~kg} / \mathrm{m}^{3} \mathrm{CEM} \mathrm{III/B}$ with a $w / c=0.44$. Each bridge element consists of 2 precast sections which were combined by a closing joint which was cast in-situ. All precast sections were produced with steam-curing for 2 hours. The bridge elements were produced and placed in 1984 .

* Corresponding author: joost.gulikers@rws.nl 


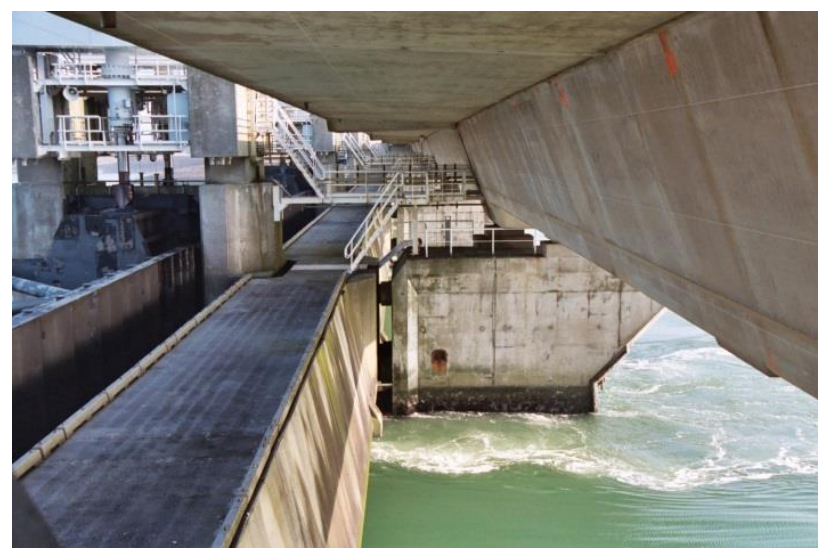

Fig. 1. View of the location and exposure conditions of the walls of the box girder bridge.

The lowest part of the bridge is located at a level of $+6 \mathrm{~m}$ above MWL whereas the bridge deck is at a height of $+11.75 \mathrm{~m}$ above MWL. All elements of the box girder bridge are T-shaped which implies that the concrete faces on the underside are sheltered from rain and other precipitation. In addition, the presence of sliding doors located in front at the North Sea side will shield a significant part of the concrete surfaces. Consequently, the environment can be described as predominantly atmospheric with exposure to chlorides contained in aerosol, and occasionally seawater spray and incidentally splash (during periods of storm when the sliding doors are lowered).

\section{Field investigations performed in 2002}

\subsection{Measured chloride ingress profiles}

At the time of the first site investigations in 2002 the effective time of exposure amounted to 18 years. In order to collect representative data on chloride ingress over the complete length of the walls of the box girder bridge, a total of 80 concrete cores were retrieved. As on the entire surface of the bridge walls, no damage was observed; the locations for core drilling were more or less arbitrarily chosen from the perspective of relative ease of accessibility. In practice, this meant that cores were drilled approximately halfway up the height of the walls. With respect to the windward exposure side and gravel concrete bridge elements, 13 cores were drilled. Upon drilling, the cores were sent to a laboratory for chemical analysis yielding quantitative information on chloride ingress based on depth intervals of $10 \mathrm{~mm}$. These investigations result in a number of chloride penetration profiles providing information on the current electrochemical state of the reinforcing bars and the depth dependent profile may then be used for prediction of future ingress. Fig. 2 shows the results on chloride ingress profiles measured on 13 cores retrieved from 7 different bridge elements.

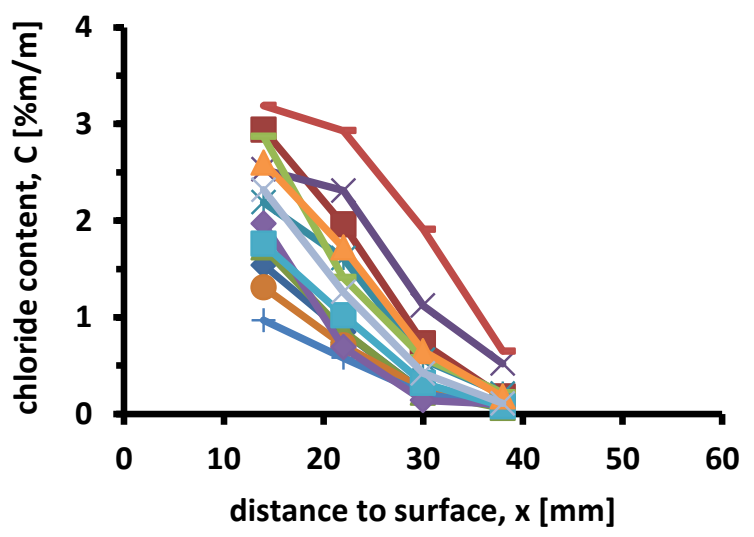

Fig. 2. Measured chloride profiles (2002) in the walls of the box girder bridge after 18 years of exposure (windward side).

It can clearly be seen that the scatter in chloride ingress is significant, despite the uniform concrete quality and exposure conditions, even at the level of the reinforcing steel $(\mathrm{c}=40 \mathrm{~mm})$, with chloride contents ranging from $0.05 \%$ to $0.80 \%$ ( $\mathrm{m} / \mathrm{m}$ cement). Generally, for chloride levels in excess of $0.7 \%$ the probability of depassivation of reinforcing steel is considered high, see Fig. 7 [3]. This conclusion resulted in great concern of the local asset owner on both the actual and future condition of the box girder bridge in view of timely and effective interventions to be considered.

\subsection{Prediction of time to depassivation}

In order to obtain quantitative information on the progress of chloride ingress and the resulting depassivation of the steel reinforcement over time, the measured chloride profiles were subjected to regression analysis to obtain best-fit values for relevant parameters, using an analytical solution to Fick's $2^{\text {nd }}$ law of diffusion, commonly referred to as the error function solution, as the chloride ingress model:

$$
C(x, t)=C_{s}-\left(C_{s}-C_{i}\right) \cdot \operatorname{erf}\left(\frac{x}{2 \sqrt{D_{a} \cdot t}}\right)
$$

where $\mathrm{x}$ is the distance to the exposed concrete surface, and $t$ indicates either the age of the concrete or to the effective exposure time, whereas erf refers to the error function.

In fact, this expression contains 3 unknown parameters, i.e. the surface chloride content, $\mathrm{C}_{\mathrm{s}}$; the initial chloride content, $\mathrm{C}_{\mathrm{i}}$; and the apparent chloride diffusion coefficient, $D_{a}$. Fig. 3 shows the best-fit chloride profiles resulting from this regression exercise, using the least square method. 


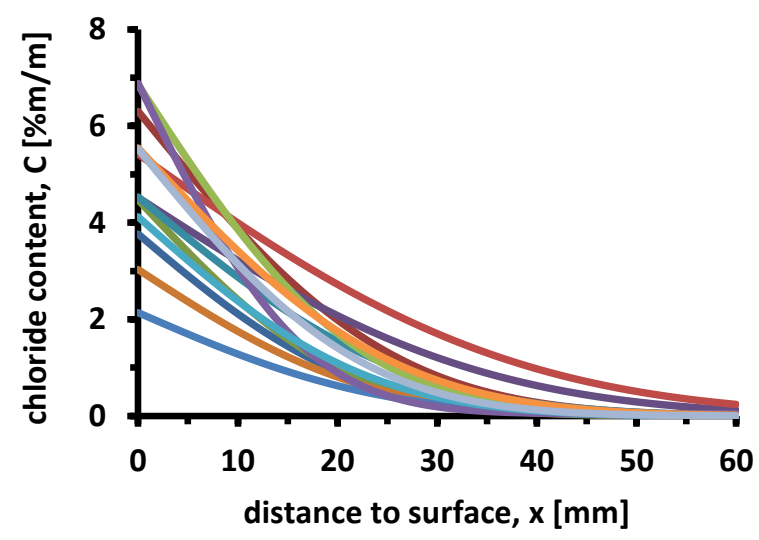

Fig. 3. Fitted chloride profiles (2002) in the walls of the box girder bridge after 18 years of exposure (windward side).

The values of the fitted surface chloride contents, $\mathrm{C}_{\mathrm{s}, \mathrm{fit}}$, ranged from 2.1 to $6.9 \%(\% \mathrm{~m} / \mathrm{m}$ cement $)$, whereas the values for the initial chloride content, $\mathrm{C}_{\mathrm{i}, \mathrm{fit}}$, generally amounted to $0 \%$ and incidentally up to $0.06 \%$. This level of $\mathrm{C}_{\mathrm{i}}$ was as expected as no chlorides were allowed to be mixed in during concrete production. If significant levels for the chloride content at depths $>50 \mathrm{~mm}$ had been found then this could hint at a systematic bias in the chloride measurements. The apparent chloride diffusion coefficient, $\mathrm{D}_{\mathrm{a}, \mathrm{fit}}$, was found to range between 4.7 to $24.7 \mathrm{~mm}^{2} / \mathrm{yr}$, corresponding to $0.15 \cdot 10^{-12} \mathrm{~m}^{2} / \mathrm{s}$ and $0.78 \cdot 10^{-12} \mathrm{~m}^{2} / \mathrm{s}$, respectively, with an average of $11.2 \mathrm{~mm}^{2} / \mathrm{s}\left(0.35 \cdot 10^{-12} \mathrm{~m}^{2} / \mathrm{s}\right)$. These values for $\mathrm{D}_{\mathrm{a}, \mathrm{fit}}$, as determined by regression analysis, for a concrete age of 18 years are indicative for a very dense concrete cover zone. This qualification is supported by other visual and microscopic observations on the concrete cores.

Adopting a conservative approach by assuming that the diffusion coefficient $\mathrm{D}_{\mathrm{a}}$ remains constant over time and $\mathrm{C}_{\text {crit }}=0.5 \%$ for individual concrete cores onset of reinforcement corrosion would occur within -7 to +38 years.

However, for such predictions it should be borne mind that in practice it is generally observed that the chloride diffusion coefficient $D_{a}$ gradually decreases over time, and the general trend is described by an empirical relationship given by:

$$
D_{a}(t)=D_{a}\left(t_{r e f}\right) \cdot\left(\frac{t_{\text {ref }}}{t}\right)^{n}
$$

where $n$ is the so-called ageing factor and $t_{\text {ref }}$ is the reference age at which the reference value $D_{a(\text { tref })}$ is determined (from measured chloride profiles).

Moreover, there is significant uncertainty on the actual level of the critical chloride content, $\mathrm{C}_{\text {crit. }}$. According to [3] $\mathrm{C}_{\text {crit }}$ demonstrates a large variation which calls for a probabilistic approach.

An important point for the local asset manager is that at the time of inspection (2002), nowhere could damage be observed that could be directly or indirectly linked to the presence of reinforcement corrosion. This observation raised considerable doubt on the conclusions with respect to the calculated residual service life. As a result, additional large-scale investigations were proposed to either validate or invalidate the conclusions based on the 2002 inspection results.

\section{Field investigations performed during 2005-2012}

In view of the serious concern of the local asset manager on the actual and future condition of the bridge elements with respect to corrosion of the reinforcing steel, during the time period 2005-2014 a variety of non-destructive test methods were applied, including potential mapping, galvanostatic pulse and concrete resistivity. Regarding potential mapping, large scale investigations were executed in 2010 using a small grid size as to detect any spots suspected of active reinforcement corrosion. These essentially non-destructive measurements comprised the complete underside of several bridge elements, including the external surface of both cantilevers and walls, as well as the floor, see Fig. 4.

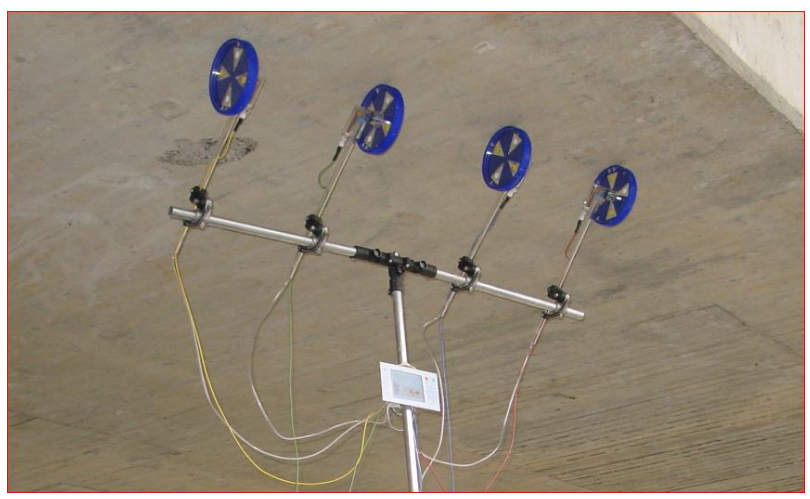

Fig. 4. Potential mapping executed on the floor of a bridge element using 4 potential wheels in parallel.

Generally, the potential values recorded were less negative than $-200 \mathrm{mV}\left(\mathrm{vs} \mathrm{Cu} / \mathrm{CuSO}_{4}\right)$ whereas resistivity measurements performed at the concrete surface generally demonstrated values well in excess of $500 \Omega \mathrm{m}$ (equal to $50 \mathrm{k} \Omega \mathrm{cm}$ ). Consequently, none of these investigations indicated locations clearly suspect of reinforcement corrosion. This interpretation was also supported by the absence of any damage on the concrete surfaces investigated.

Eventually it was decided to perform a new series of chloride profiling in 2016 to validate the results of the chloride profiling in 2002. In addition, combining the results of 2002 and 2016 would allow a more reliable prediction of the future development of chloride ingress.

\section{Field investigations performed in 2016}

In view of the importance of the box girder bridge for traffic and the preference of the asset manager for preventive rather than corrective maintenance, in 2016 a new large campaign was organised to obtain quantitative information on chloride ingress. As the previous investigations were performed in 2002 , i.e. at an age of 18 years, the information obtained at a significantly later age (32 years) would shed more light on possible ageing 
effects affecting chloride ingress over time and thus allow for a more reliable prediction of future ingress.

Prior to coring, visual inspections were performed to identify any possible locations suspected of reinforcement corrosion which could be used as preferential spots for coring, however, nowhere could damage related to corrosion be observed. In total more than 90 concrete cores were retrieved from several bridge elements, not only from the bridge walls but also from both cantilevers as well as from the bridge floor. In this paper only the data on chloride ingress in the wall on the windward side of the box girder bridge will be presented and discussed.

In contrast to the measurements executed in 2002, chloride profiling was performed at 3 different heights. In order to avoid any discussion on the method used for chloride analysis the cores were sent to the same laboratory that had performed the chloride analysis in 2002. As inspections executed prior to these investigation didn't reveal any damage on the concrete surface, the locations for coring were chosen according to pre-defined gridlines.

Chloride profiling was performed on 5 different bridge elements all made with traditional gravel concrete, comprising 18 concrete cores. The measured chloride profiles are shown in Fig. 5.

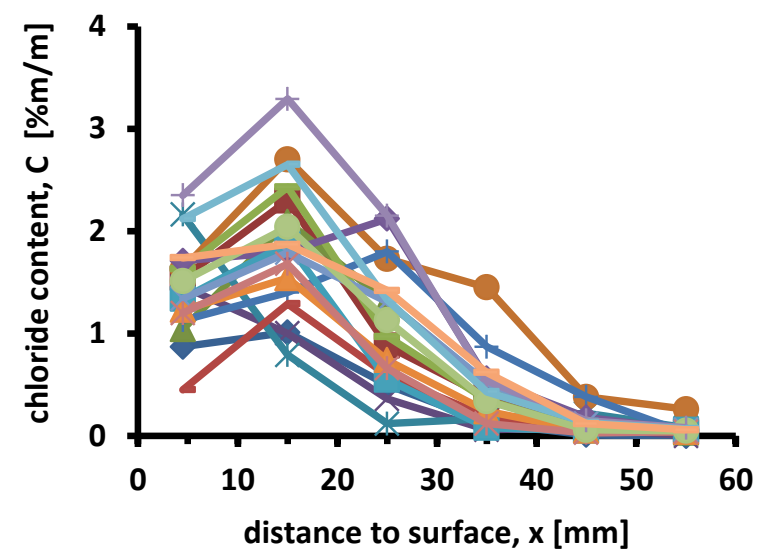

Fig. 5. Measured chloride profiles (2016) in the walls of the box girder bridge after 32 years of exposure (windward side).

With respect to onset of corrosion the chloride contents found at the level of the reinforcing steel, $\mathrm{x}=\mathrm{c}$, are relevant. According to the results of the regression analysis the chloride content at $\mathrm{x}=40 \mathrm{~mm}$ range from $0.05 \%$ to $0.84 \%$ with an average of $0.29 \%$. However, these values achieved through regression analysis are generally higher than was actually measured at depths between 35 and $55 \mathrm{~mm}$.

\section{Interpretation and prediction}

Similar to the procedure adopted for the interpretation of the results from the previous inspection (2002) all measured chloride profiles obtained in 2016 were subjected to regression analysis yielding best-fit values for the model parameters, $\mathrm{C}_{\mathrm{s}, \text { fit }}, \mathrm{C}_{\mathrm{i}, \text { fit }}$ and $\mathrm{D}_{\mathrm{a} \text {,fit, }}$, see Fig. 6 .

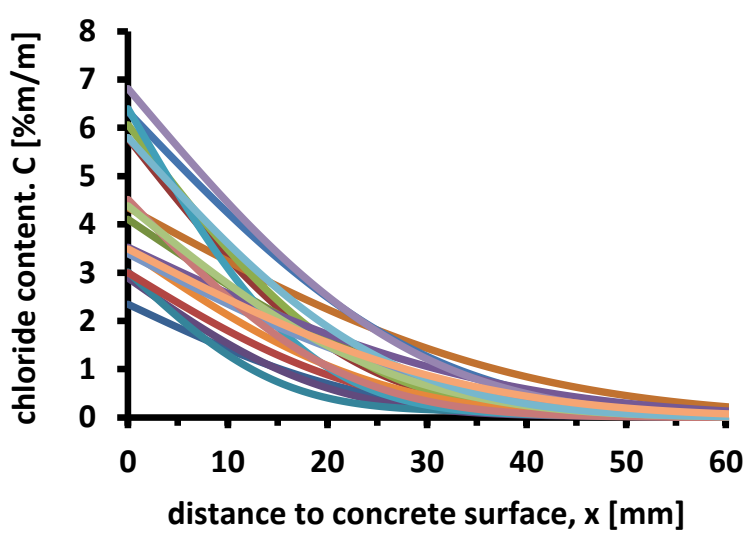

Fig. 6. Fitted chloride profiles (2016) in the walls of the box girder bridge after 18 years of exposure (windward side).

The values obtained through regression analysis for $\mathrm{C}_{\mathrm{s}, \mathrm{fit}}$ ranged from 2.3 to $6.8 \%$ with an average of $4.4 \%(\mathrm{~m} / \mathrm{m}$ cement), whereas the magnitude of $\mathrm{C}_{\mathrm{i}, \text { fit }}$ was almost 0 .

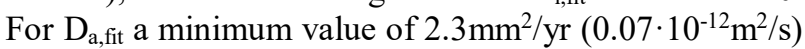
and a maximum value of $14.8 \mathrm{~mm}^{2} / \mathrm{yr}\left(0.47 \cdot 10^{-12} \mathrm{~m}^{2} / \mathrm{s}\right)$ were derived, whereas the average value amounted to $7.1 \mathrm{~mm}^{2} / \mathrm{yr}\left(0.23 \cdot 10^{-12} \mathrm{~m}^{2} / \mathrm{s}\right)$.

Comparing the average values obtained for $\mathrm{D}_{\mathrm{a}, \mathrm{fit}}$ at $\mathrm{t}_{\text {insp1 }}=18 \mathrm{yr}$ and at $\mathrm{t}_{\mathrm{insp} 2}=32 \mathrm{yr}$ the magnitude of the ageing factor was calculated to be $\mathrm{n} \approx 0.78$. This value is much higher than $\mathrm{n}=0.45$ which is reported in [3] as an average value for concrete produced with CEM III/B exposed to a marine environment.

In addition, in [4] it is advocated that a higher value for $\mathrm{C}_{\text {crit }}$ could be used for high quality concrete without showing cracks in the concrete cover. As the bridge elements are prestressed and the compressive strength exceeds $50 \mathrm{MPa}$ it seems reasonable to adopt a higher value for $\mathrm{C}_{\text {crit }}$.

In view of the uncertainties involved for each chloride profile, a probabilistic approach was adopted assuming that for an individual chloride profile both $\mathrm{C}_{\mathrm{s}, \mathrm{fit}}$ and $\mathrm{C}_{\mathrm{i} \text {,fit }}$ remain essentially constant over time, whereas the ageing factor $\mathrm{n}$ and the critical chloride content are regarded as stochastic variables.

For the ageing factor a beta distribution was used with a mean of $\mu \mathrm{n}=0.75$ and a standard deviation, $\sigma \mathrm{n}=$ 0.05 . For the lower and upper boundaries, a minimum a $=0.0$ and a maximum $\mathrm{b}=1.0$ were chosen. For the critical chloride content, a beta distribution was applied with $\mu \mathrm{C}_{\text {crit }}=0.75 ; \sigma \mathrm{C}_{\text {crit }}=0.15 ; \mathrm{a}=0.20 \%$ and $\mathrm{b}=$ $2.00 \%$, see Fig. 7.

The results of these probabilistic calculations for each chloride profile, using the modified statistical values of $n$ and $\mathrm{C}_{\text {crit, }}$, identified as "realistic scenario", are shown in Fig. 8. For comparison Fig. 9 shows the results obtained according to the "fib 34 scenario" when the values according to [3] would have been adopted (n: $\mu \mathrm{n}$ $=0.45 ; \sigma \mathrm{n}=0.20$ and $\mathrm{C}_{\text {crit }}: \mu \mathrm{C}_{\text {crit }}=0.60 \% ; \sigma \mathrm{C}_{\text {crit }}=$ $0.15 \%)$. 


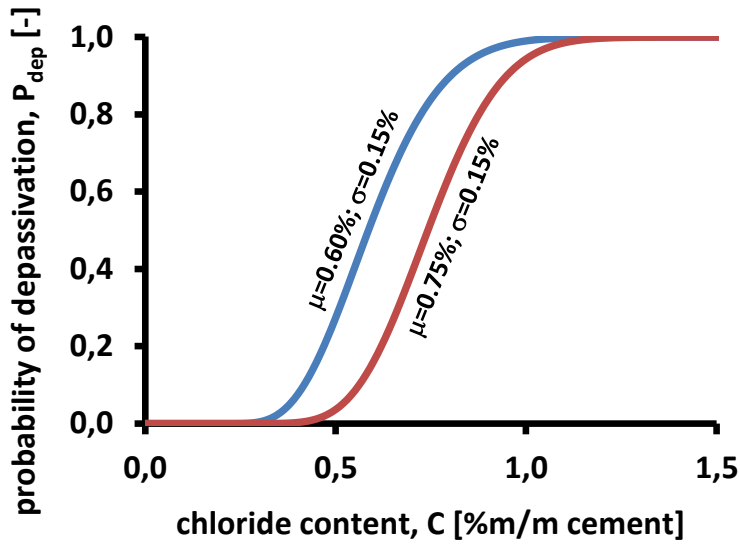

Fig. 7. Distribution of the probability of depassivation for the critical chloride content as adopted in the probabilistic calculations.

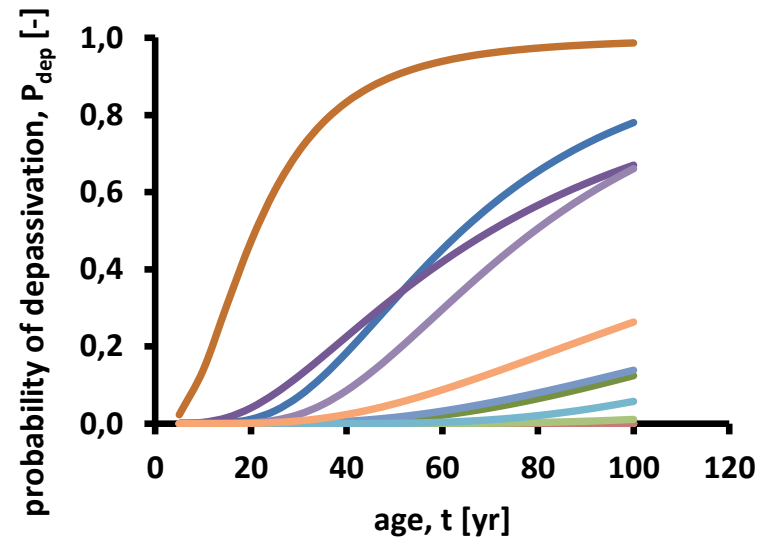

Fig. 8. Development of probability of depassivation for individual chloride profiles according to input values adopted for the "realistic scenario".

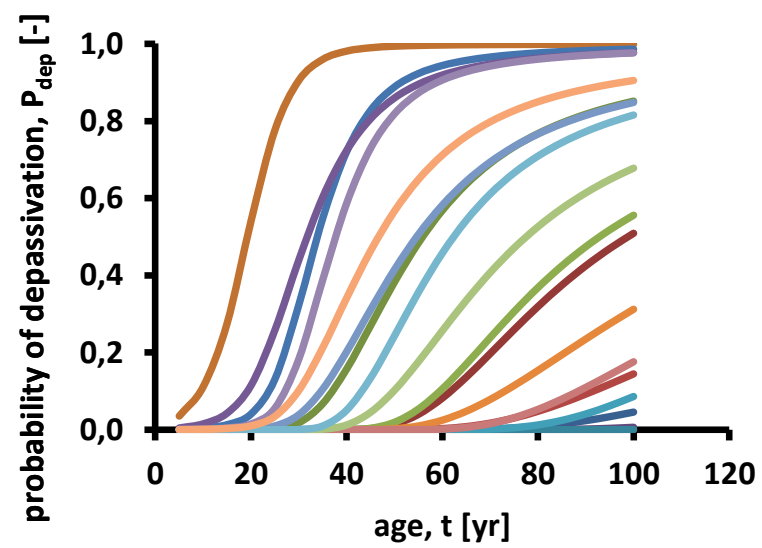

Fig. 9. Development of probability of depassivation for individual chloride profiles according to input values for the "fib 34 scenario".

However, even for the results according to the "realistic scenario", the conclusion of these predictive calculations is that localised onset of corrosion may start within less than a decade after the second inspection in 2016. On the other hand, in the area of the worst chloride profile found in the 2016 inspections no evidence of active reinforcement corrosion was found, although the probabilistic calculations resulted in a $78 \%$ probability of depassivation being present.

\section{Discussion and outlook}

During the time period 2002-2016 extensive investigations have been performed with respect to chloride ingress into the wall of a number of bridge elements. Although it has been established that the concrete cover is of high quality with very low permeability, as supported by the very low values for the apparent diffusion coefficient $D_{a}$, locally, chloride ingress has advanced quite deep. However, visual inspections and surface hammering have not identified any spots that are suspected of reinforcement corrosion.

As an explanation for the contrasting results of visual inspection on the one hand and chloride content present at the level of the reinforcing steel and the probabilistic predictions on the other, 2 major hypotheses can be established.

The first hypothesis is that the steel-concrete interface does not show any major defects which may act as preferential sites for depassivation. The complete circumference of all reinforcing steel bars is covered with a dense and adhering cement layer without weak areas. As the bridge elements are prestressed, cracking in the concrete cover is largely absent and under normal service conditions the tensile stresses in the reinforcing bars are low. Consequently, the bond between steel bars and surrounding concrete will be hardly impaired. According to this reasoning, the critical chloride content is likely to be much higher than would normally be assumed, e.g. according to the values given in [3]. Moreover, values for $\mathrm{C}_{\text {crit }}$ may be higher for concrete produced with blast furnace slag cement (slag content $>$ $50 \%$ ). It is well known that slag cements are able to bind more chlorides, thus the concentration of free chlorides dissolved in the pore solution to attack the passive layer will be less.

The second hypothesis is based on the high concrete quality which is reflected in a high concrete resistivity even in a continuously wet exposure environment. In the investigations performed in 2002, concrete resistivity was measured on a limited number of concrete cores retrieved from the walls. Generally, the resistivity values were in excess of $500 \Omega \mathrm{m}$. This was confirmed by measurements performed on site using the 4-point Wenner probe placed on the wetted concrete surface of the walls. As corrosion is an electrochemical process, in concrete demonstrating resistivities in excess of $500 \Omega \mathrm{m}$ the corrosion rate is generally found to be very low [5]. This implies that the production of electrons released in the anodic reaction will be limited which explains why the potential values that have been measured are corresponding to a passive state.

Given the high uncertainties in the input values of the most dominant model parameters it was decided not to rely on predictions based on numerical modelling. Meanwhile in practice a probabilistic approach is often advocated to take into account these uncertainties, however, experience, with this paper as an example, has 
shown that such an approach eventually turns out to become a "numbers game" to disguise the lack of knowledge and responsibility.

The results obtained from the investigations executed in 2002 and 2016 have provided a better impression of the development of chloride ingress over time. However, the chloride profiles that have been measured show a significant scatter, despite the fact that both the quality of the concrete cover and the environmental load with respect to moisture and chlorides is considered quite uniform over the exposed concrete surface of the wall. Consequently, it is concluded that the spatial variability of chloride ingress is considerable, even for this relatively ideal situation. This implies that a considerable number of cores would be required to have a reliable impression of chloride ingress over the complete wall surface.

At present the local asset manager is tired of all these investigations resulting in more confusion on the actual condition of the box girder bridge with respect to reinforcement corrosion. Therefore, in the near future, no further investigations on site are envisaged but a pragmatic engineering approach based on common sense will be adopted.

\section{References}

1. CUR, Publication 215, Durability of marine concrete structures, 2005 (in Dutch)

2. DuraCrete, Final Technical Report, Document BE95-1347/R17 (2000)

3. fib, Model code for service life design, bulletin 34, 2006

4. M. Breit, C. Dauberschmitt, C. Gehlen, C. Sodeikat, A. Taffe, U. Wiens, Approach on the critical chloride content in reinforced concrete structures, Beton- und Stahlbetonbau, 106 (2011), Heft 5 (in German)

5. P. Langford, J. Broomfield, Monitoring the corrosion of reinforcing steel, Construction Repair, $1987,1(2)$ 